Draft Version SEPTEMber 8, 2021

Preprint typeset using $\mathrm{LATEX}_{\mathrm{E}}$ style emulateapj v. 5/2/11

\title{
THE COSMIC ABUNDANCE OF CLASSICAL MILKY WAY SATELLITES
}

\author{
LOUis E. Strigari AND Risa H. WEChSLER \\ Kavli Institute for Particle Astrophysics and Cosmology, Physics Department, Stanford University, Stanford, CA 94305 USA \\ Draft version September 8, 2021
}

\begin{abstract}
We study the abundance of satellites akin to the brightest, classical dwarf spheroidals around galaxies similar in magnitude and isolation to the Milky Way and M31 in the Sloan Digital Sky Survey. From a combination of photometric and spectroscopic redshifts, we bound the mean and the intrinsic scatter in the number of satellites down to ten magnitudes fainter than the Milky Way. Restricting to magnitudes brighter than Sagittarius, we show that the Milky Way is not a significant statistical outlier in its population of classical dwarf spheroidals. At fainter magnitudes, we find an upper limit of 13 on the mean number of satellites brighter than the Fornax dwarf spheroidal. Methods to improve these limits that utilize full photometric redshift distributions hold promise, but are currently limited by incompleteness at the very lowest redshifts. Theoretical models are left to explain why the majority of dark matter subhalos that orbit Milky Way-like galaxies are inefficient at making galaxies at the luminosity scale of the brightest dwarf spheroidals, or why these subhalos predicted by $\Lambda$ CDM do not exist.
\end{abstract}

Subject headings: dark matter — galaxies: dwarf — galaxies: formation — halo — Local Group

\section{INTRODUCTION}

Around the Milky Way (MW) Galaxy orbit nearly two dozen known satellite galaxies that have a range of magnitudes down to twenty times fainter than the MW itself. The two brightest satellites, the Large Magellanic Cloud (LMC) and the Small Magellanic Cloud (SMC), are approximately two and four magnitudes fainter than the MW, respectively. Numerical simulations indicate that there is a $\lesssim 10 \%$ chance that a $10^{12} M_{\odot}, \mathrm{MW}$ mass dark matter halo hosts two satellites as massive as the Magellanic Clouds (Bovlan-Kolchin et al. 2010; Busha et al. 2011). From an observational perspective, analysis of galaxies from the Sloan Digital Sky Survey Seventh Data Release (SDSS DR7) indicates that MW magnitude-galaxies have two galaxies as bright as the Magellanic clouds only $\sim 5 \%$ of the time, and on average have $\sim 0.3$ satellites within four magnitudes of the MW (Liu et al. 2011; Guo et al. 2011; Lares et al. 2011; Tollerud et al. 2011). Thus the MW is somewhat atypical in having two satellites with this luminosity difference, but the probability of having a given number at this scale is in excellent agreement between theory and observation (see further discussion in Busha et al. 2011).

Beyond the Magellanic Clouds (MCs), the brightest satellites are the Sagittarius, Fornax, and Leo I dwarf spheroidals (dSphs), approximately six, eight, and nine magnitudes fainter than the MW, respectively. Though at present challenging, a measurement of the abundance of satellite galaxies as bright as these classical dSphs is important for several reasons. From the observational perspective, it improves our understanding of the MW and its place on a cosmic scale, providing information on the number of bright satellites around other galaxies over a regime in which we are believed to be complete around the MW (Walsh et al. 2009), in particular away from the Galactic plane (Klevna et al. 1997; Willman 2010). From the theoretical perspective, understanding the abundance of classical dSphs is important because these objects reside in the least massive dark matter halos that contain visible light, corresponding to halo mass scales at which various processes including supernova feedback effects and suppression of gas accretion from reionization becomes important Bullock et al. 2000; Benson et al. 2002; Somerville 2002).

Though the above processes certainly affect the formation of dSphs, theoretical models that include them have still found it challenging to match both the distribution of luminosities of the bright MW satellites and their kinematic properties (Font et al. 2011). Estimates of the luminosity function down to scales of the classical dSphs predict that they reside in dark matter halos with velocity dispersions $\gtrsim 20 \mathrm{~km} / \mathrm{s}$ (Cooper et al. 2010). These models typically predict tens of satellites brighter than Fornax, $\sim 10^{7} L_{\odot}$. Both of these predictions are in tension with observations of the MW satellite population. On the one hand, the predicted velocity dispersion for bright satellites is nearly two times larger than the observed $\sim 10 \mathrm{~km} / \mathrm{s}$ velocity dispersion of bright dSphs. On the other hand, since the observational sample is complete for objects with reasonable surface brightness with luminosity $\gtrsim 10^{7} L_{\odot}$, it is difficult to invoke that a population of objects this luminous has been missed by observations.

More detailed analysis of dSph kinematics indicates that their maximum circular velocities are $\lesssim 30 \mathrm{~km} / \mathrm{s}$ (Strigari et al. 2010). In particular, even though it is very bright, Fornax has a strongly constrained maximum circular velocity at a relatively low $\sim 20 \mathrm{~km} / \mathrm{s}$. Matching these results with numerical simulations of the Galactic satellite population in a $\Lambda \mathrm{CDM}$ cosmology (Springel et al. 2008; Diemand et al. 2008) indicates that on average there should be $\sim 25-75$ of dark matter satellites with maximum circular velocity greater than that of Fornax, that are either too faint to be detected in surveys or devoid of baryonic material entirely (Boylan-Kolchin et al. 2011).

The above results indicate that from a combination of observations and theory, the classical problem of the 
abundance of satellites within $\Lambda$ CDM can be boiled down to a "Fornax problem": more specifically, why is it that for an observed galaxy at the luminosity scale of $\sim 10^{7} L \odot$, there are scores of dark subhalos that have the same dark matter mass, but apparently no stars in them at all? There are a few outstanding ideas that remain to answer this question. First, the tension may point directly to severe inefficiency and stochasticity of galaxy formation at the dark matter halo mass scale of the dSphs. Second, it may be that there are required modifications to the current sample of numerical simulations: in particular, baryons may significantly modify the dark matter distributions in satellites (e.g. Wadepuhl \& Springel (2011); Parry et al. (2011); di Cintio et al. (2011)), or there may even be a necessary modification to the cosmological model (Lovell et al. 2011). The third idea is perhaps the most straightforward of all; namely, that the MW is rare amongst galaxies of its kind, a result of a rare downward fluctuation in its population of bright satellites.

In this paper, we address for the first time this latter issue of the distribution of satellites as bright as the classical dSphs around MW-analog galaxies. We construct an observational sample of spectroscopic galaxies like the MW using SDSS, and search for faint satellites using SDSS DR8 imaging data and photometric redshift probability distributions. At the faintest end, we place an upper limit on the number of satellites down to the magnitude scale of the Leo I dSph. At the brightest end, we find an average of $\lesssim 2$ objects brighter than Sagittarius. Our results imply that, in terms of its bright dSph satellite population, the MW does not stand out as a significant statistical outlier.

\section{DATA}

Our analysis begins by selecting MW-analog galaxies, which is similar to the analysis presented in Liu et al. (2011). We refer to these galaxies as primaries. We use a magnitude for the MW of $M_{V}=-20.9$ van den Bergh 2000), which corresponds to $M_{\mathrm{r}}=-20.4$ (see Liu et al. 2011, for discussion), and we consider primaries within \pm 0.25 magnitudes of this value. We consider a second set of slightly brighter galaxies, with $M_{\mathrm{r}}=-20.7$, for comparison with M31. Primary galaxies are selected from the NASA-Sloan Atlas (v0_1_1) data for nearby galaxies $(z<0.055)$ with more complete spectroscopy and imaging from GALEX. We limit our analysis to isolated galaxies that have no galaxy with a luminosity equal to or greater than the MW or M31 within $0.4 \mathrm{Mpc}$ (following the same methods described in Liu et al. 2011). We make a few cuts to exclude very nearby galaxies which may have incorrect photometry, specifically those with $r<11$, SIZE $<5 *$ SERSIC_TH50, or with $g-r<0$.

To perform the search for satellites around MW and M31-analogs, we utilize the both DR8 imaging and spectroscopic catalog (Aihara et al. 2011). We use the galaxy catalog and the photometric redshifts probability distributions from Sheldon et al. (2011), which is complete for $r<21.8$. The spectroscopic sample is complete for $r<17.77$. We exclude primary galaxies for which the DR8 photometry is incomplete within $250 \mathrm{kpc}$ of the

\footnotetext{
1 http://www.nsatlas.org/data
}

primary (using a mask kindly provided by Erin Sheldon; the motivation for this cut will become more evident in Sec. 3).

For a given magnitude difference from the primary, $\Delta m$, and satellite absolute magnitude threshold, Table 1 provides the corresponding apparent $r$-band magnitude threshold and the number of primary galaxies. Columns 4-6 correspond to our photometric sample, while columns 7-9 correspond to our spectroscopic sample. The meaning of these samples will become more clear from our discussion in Sec. 3. For the photometric sample of MWanalog primaries, we have explicitly included $\Delta m=7.7$, corresponding to the Fornax dSph. In comparison to the sample of Liu et al. (2011), our sample with a threshold cut at the magnitude of the brightest satellites, i.e. the MCs, is smaller because we restrict to more nearby galaxies in the NASA-Sloan Atlas $(z<0.055)$. Since the primary focus of our analysis is on the faintest satellites, our conculsions are insensitive to the smaller sample of very bright satellites.

\section{METHODS}

Consider a galaxy that has been selected as a MW or M31-analog via aforementioned methods. We define the "signal" region as circular area centered around the primary, corresponding to a physical radius $R$ at the redshift of the primary. For the main analysis in this paper we take a $R=250 \mathrm{kpc}$ for the signal region, corresponding to the approximate viral radius of the MW and M31 (Springel et al. 2008; Diemand et al. 2008). In the signal region we search for galaxies that are between two magnitudes fainter and a threshold of $\Delta m$ magnitudes fainter than the primary. For the $\imath^{t h}$ primary, we label the number of galaxies in the signal region as $n_{t, \imath}$. For comparison to the number of counts within the signal region, we associate with each primary a background region. The background region is an annulus with an inner radius $R$, and an outer radius chosen to enclose the same area as the signal region. We label the number of galaxies within the background region associated with the $\imath^{\text {th }}$ primary as $n_{b, \imath}$. A local estimation of the background via an annulus connected to the signal region has been shown to be unbiased, even accounting for galaxy clustering (Chen et al. 2006; Liu et al. 2011; Lares et al. 2011; Guo et al. 2011).

We are interested in obtaining an estimate for the mean number of satellites around MW and M31-like galaxies, given the measurements of $n_{t, \imath}$ and $n_{b, \imath}$ around a large sample of primaries. We obtain three separate estimates for the combination of $n_{t, \imath}$ and $n_{b, \imath}$, which differ both in the cuts that are made on the data sample and the method in which the sample is obtained. The first estimate uses spectroscopic redshift information for the satellites, while the second and third methods use photometric redshifts. We now detail specifically how each of these estimates are obtained in turn.

\subsection{Method 1: Spectroscopic satellites}

Our first method for estimating $n_{t, \imath}$ and $n_{b, \imath}$ uses spectroscopic redshifts for both primaries and satellites. Since the DR8 sample is spectroscopically-complete down to $r<17.77$, we are able to estimate the number of satellites brighter than $\Delta m=6,5,4$. As indicated in Table 1, these magnitude differences correspond to primary 
Table 1

Properties of primary galaxies for each magnitude limit. The upper eight rows correspond to Milky Way-like primaries, the bottom seven rows correspond to M31-like primaries.

\begin{tabular}{|c|c|c|c|c|c|c|c|c|}
\hline \multirow[b]{2}{*}{ Sample } & \multirow[b]{2}{*}{$\Delta m$} & \multirow[b]{2}{*}{$\begin{array}{c}\text { Satellite Absolute } \\
\text { Magnitude }\end{array}$} & \multicolumn{3}{|c|}{ Photometric } & \multicolumn{3}{|c|}{ Spectroscopic } \\
\hline & & & $\begin{array}{c}\text { Primary Apparent } \\
\text { Magnitude }\end{array}$ & $\begin{array}{l}\text { Number of } \\
\text { Primaries }\end{array}$ & $\begin{array}{l}\text { Mean Number } \\
\text { of Satellites }\end{array}$ & $\begin{array}{c}\text { Primary Apparent } \\
\text { Magnitude }\end{array}$ & $\begin{array}{l}\text { Number of } \\
\text { Primaries }\end{array}$ & $\begin{array}{l}\text { Mean Number } \\
\text { of Satellites }\end{array}$ \\
\hline \multirow[t]{8}{*}{ MW } & 10 & -10.9 & 11.8 & 49 & $<400$ & - & - & - \\
\hline & 9 & -11.9 & 12.8 & 148 & $<100$ & - & - & - \\
\hline & 8 & -12.9 & 13.8 & 727 & $<30$ & - & - & - \\
\hline & 7.7 & -13.2 & 14.1 & 1117 & $<13$ & - & - & - \\
\hline & 7 & -13.9 & 14.8 & 2727 & $3.10 \pm 1.60$ & - & - & - \\
\hline & 6 & -14.9 & 15.8 & 7574 & $1.50 \pm 0.47$ & 11.8 & 49 & $1.42 \pm 0.56$ \\
\hline & 5 & -15.9 & 16.8 & 8388 & $0.92 \pm 0.26$ & 12.8 & 148 & $0.62 \pm 0.18$ \\
\hline & 4 & -16.9 & 17.8 & 8388 & $0.51 \pm 0.15$ & 13.8 & 727 & $0.25 \pm 0.05$ \\
\hline \multirow{7}{*}{ M31 } & 10 & -11.2 & 11.8 & 41 & $<300$ & - & - & - \\
\hline & 9 & -12.2 & 12.8 & 151 & $<60$ & - & - & - \\
\hline & 8 & -13.2 & 13.8 & 834 & $<13$ & - & - & - \\
\hline & 7 & -14.2 & 14.8 & 3370 & $2.41 \pm 1.55$ & - & - & - \\
\hline & 6 & -15.2 & 15.8 & 7427 & $1.31 \pm 0.49$ & 11.8 & 41 & $1.01 \pm 0.54$ \\
\hline & 5 & -16.2 & 16.8 & 7432 & $1.02 \pm 0.47$ & 12.8 & 151 & $0.37 \pm 0.12$ \\
\hline & 4 & -17.2 & 17.8 & 7432 & $0.50 \pm 0.21$ & 13.8 & 834 & $0.20 \pm 0.04$ \\
\hline
\end{tabular}

apparent magnitudes $r<11.8,12.8,13.8$, respectively. Specifically we find 49,148 , and 727 primaries that satisfy $r<11.8,12.8,13.8$, respectively. For a given set of primaries, we select galaxies in the spectroscopic sample that satisfy two criteria. First, as described above we determine those galaxies in the signal region that satisfy $R<250 \mathrm{kpc}$, as well as galaxies within the corresponding background annulus. Second, we impose a cut so that both the galaxies in the signal region and the galaxies in the background region lie within a redshift $\Delta z=0.001$ $\left(300 \mathrm{kms}^{-1}\right)$ of the primary. This redshift cut is appropriate when accounting for both the expected physical size of the dark matter halos of the primaries, and for redshift space distortions.

Using spectroscopic redshifts to identify satellites clearly reduces background contamination from galaxies at vastly different redshifts than the primary. However, the obvious downside is that, when demanding the satellites have a spectroscopic redshift, the sample of primaries is much smaller than the corresponding sample of primaries obtained by only demanding complete photometry for the satellites. Further, when restricting to spectroscopic satellites we run out of very bright primaries, meaning we are unable to probe very faint magnitude differences, $\Delta m>6$, that are the main goal of this work.

\subsection{Method 2: Full photometric redshift distributions}

For our second estimate of $n_{t, \imath}$ and $n_{b, \imath}$, we use the photometric galaxy sample of Sheldon et al. (2011), which is complete down to $r<21.8$. In this case, we are able to estimate the number of satellites down to magnitudes as faint as $\Delta m=10$, corresponding to primaries with $r<11.8$. The total number of primaries for each $\Delta m$ is shown under the photometric heading in Table 1.

For each galaxy with $r<21.8$, Sheldon et al. (2011) assign a probability, $p(z)$, that it resides in one of $N_{p h o t}=$ 35 redshift bins. Method 2 specifically uses the information in these $p(z)$ distributions. With these distributions in hand, again we start by considering a single primary galaxy. We use the same redshift bins in which the $p(z)$ 's are calculated, and from these locate the redshift bin that contains the primary. As described above we again search for galaxies within the signal region $R<250 \mathrm{kpc}$. Given this set of galaxies in the signal region, we then sample each of their corresponding discrete $p(z)$ distributions. This provides us with a redshift for each galaxy within the signal region. From a single sampling of each $p(z)$ distribution, we determine the total number of galaxies that fall into the redshift bin that contains the primary. To obtain a statistically robust estimate of the total signal and background probability distributions, we repeat the sampling procedure $\sim 100$ times to obtain the mean number of counts in the signal region in the same redshift bin as the primary, $\left\langle n_{t, \imath}\right\rangle$. Then following a similar reasoning as above, we repeat this procedure for the annular background region to obtain the corresponding mean number of counts in the redshift bin associated with the background for each primary, $\left\langle n_{b, \imath}\right\rangle$.

As long as the photometric redshift distribution for each galaxy is unbiased, the estimate of the number of satellites obtained from this method is expected to be just as accurate as the estimate that uses spectroscopic redshifts. However, systematics in photometric redshift information may bias the results. Of particular relevance is the fact that the training set used in Sheldon et al. (2011) to obtain the photometric redshifts may not have a fully representative sample of faint, low redshift galaxies. This is precisely the sample of galaxies that we are interested in identifying as satellites. If the training sample is underrepresented at low redshift, this will likely lead to a biased $p(z)$ estimate for potential satellites, and underestimate of the satellite number associated with low redshift hosts. As shown below, a comparison of the results obtained from this method to those obtained from the spectroscopic sample (method 1), over the regimes in which both are complete, may provide an estimate of the magnitude regime in which photometric redshifts are not representative for faint, low redshifts galaxies.

\subsection{Method 3: Mean photometric redshifts}

Our third method to estimate $n_{t, \imath}$ and $n_{b, \imath}$ also uses information contained in the photometric redshift distributions. However, rather than directly sampling the full $p(z)$ distributions as above, this method simply uses the information contained in the mean of these distributions. Once a mean photometric redshift is assigned to a galaxy, 


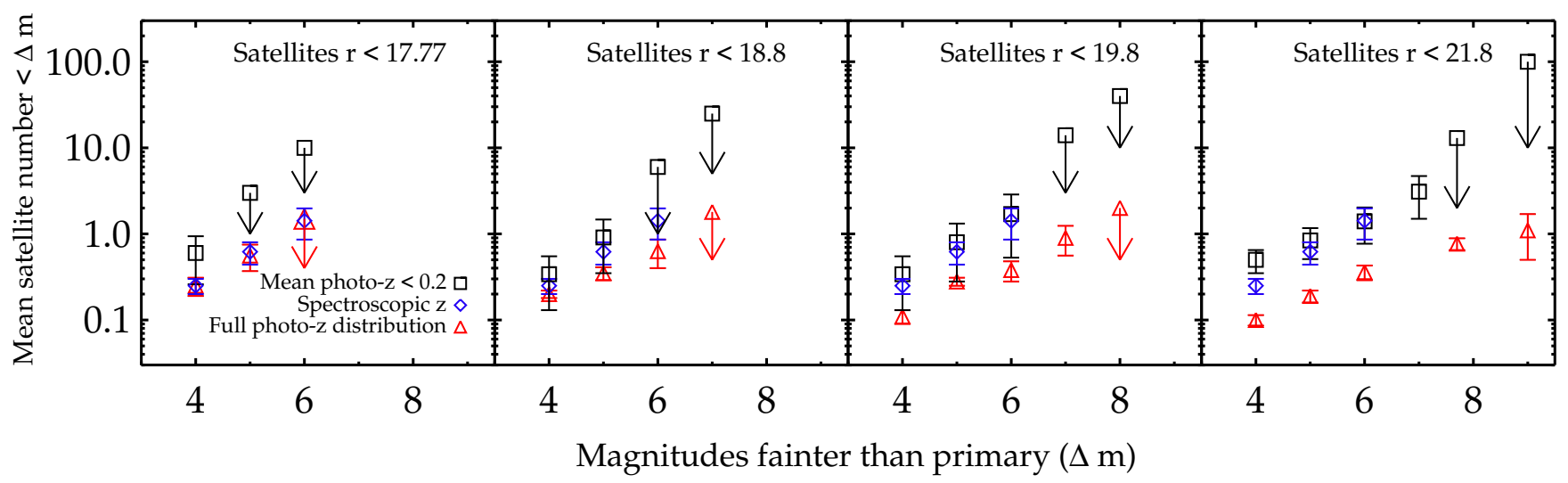

Figure 1. Mean number of satellites within $\Delta m$ of MW-like primaries, using the three different methods described in Sec. 3 In all panels, blue diamonds include only satellites with spectroscopic redshift within $\Delta z=0.001$ of the primary $(\operatorname{method} 1)$, and red triangles sample the full photometric redshift probability distributions (method 2). In the three left panels, black squares only include satellites with mean photometric redshift less than 0.2 , while in the right-most panel black squares only include satellites with mean photometric redshift less than 0.5 (method 3 ). In each of the four panels, the magnitude limit on the population of satellites from the photometric analyses (methods 2 and 3) are indicated. In all panels, upper limits are indicated as downward arrows.

implementation of this analysis method boils down to determining an appropriate redshift cut on galaxies included in the signal and background region.

To motivate the appropriate redshift cut, define the mean photometric redshift for a galaxy as

$$
\left\langle z_{p}\right\rangle \equiv \sum_{\imath=1}^{N_{p h o t}} z_{\imath} p\left(z_{\imath}\right)
$$

where $p\left(z_{\imath}\right)$ is the probability for the $\imath^{t h}$ redshift bin. Though it is simple, ascribing to a galaxy a single redshift based upon Eq. 11 will typically overestimate the true redshift of the galaxy. More specifically we are interested in the mean photometric redshifts for satellites that span the redshift range of our primary galaxies; this corresponding redshift range is 0.055. Examining the faintest sample of training set galaxies, $20.8<r<21.8$, over this redshift range of the primaries, we find that the ratio of the mean photometric redshift to the true redshift, $z_{\text {true }}$, spans a wide range $\left\langle z_{p}\right\rangle / z_{\text {true }} \sim 3-30$. Further, even for the brightest sample of training set galaxies fainter than the spectroscopic completeness limits, $17.8<r<18.8$, we find $\left\langle z_{p}\right\rangle / z_{\text {true }} \sim 1-10$.

In spite of the fact that typically $\left\langle z_{p}\right\rangle / z_{\text {true }} \gtrsim 1$, it is still possible to use the information on the mean photometric redshifts, provided that we place an appropriate upper cut on the mean photometric redshift of a galaxy that is allowed in our sample. In other words, we must estimate the number of true satellites that are lost from the sample when including galaxies less than a given $\left\langle z_{p}\right\rangle$.

We obtain this estimate for the loss fraction by again considering training set galaxies over the redshift range spanned by the set of primary galaxies of interest. Specifically, out to the maximum primary redshift, we examine the distribution of $\left\langle z_{p}\right\rangle$ for training set galaxies that are brighter than a given apparent magnitude. Then from this distribution we determine the value of $\left\langle z_{p}\right\rangle_{90}$, which we define as the redshift below which $90 \%$ of the galaxies reside. We determine $\left\langle z_{p}\right\rangle_{90}$ for each apparent magnitude threshold cut. For example we find that when searching for satellites down to $r<21.8,\left\langle z_{p}\right\rangle_{90}=0.5$. Further, we find that for $r<19.8,\left\langle z_{p}\right\rangle_{90}=0.2$. These are the cuts that we utilize in our analysis below. Because of the large sample of primaries available when using the photometric catalogue, we find that the results of method 3 are generally insensitive to the chosen value for $\left\langle z_{p}\right\rangle_{90}$.

\subsection{Likelihood}

With the methods outlined for determining the data sample of galaxies in the signal and background regions, it remains to construct the likelihood for the data. For a total sample of $n_{p}$ primaries, the mean number of satellites is the difference between the mean of the signal and background counts,

$$
\mu_{s}=\frac{1}{n_{p}} \sum_{\imath=1}^{n_{p}} n_{t, \imath}-\frac{1}{n_{p}} \sum_{\imath=1}^{n_{p}} n_{b, \imath}=\mu_{t}-\mu_{b} .
$$

We define the variance of the signal and background distributions as $\sigma_{t}^{2}$ and $\sigma_{b}^{2}$. Assuming that the number of galaxies around a primary and the background are uncorrelated, the variance is $\sigma_{s}^{2}=\sigma_{t}^{2}-\sigma_{b}^{2}$. Defining the parameter set that we estimate from the data as $\vec{x}=\left[\mu_{s}, \sigma_{s}, \mu_{b}, \sigma_{b}\right]$, for a given magnitude threshold $\Delta m$, the probability for the mean and variance is

$$
\begin{aligned}
P(\vec{x} \mid \Delta m) \propto & \prod_{\imath=1}^{n_{p}} \frac{1}{\sqrt{\sigma_{s}^{2}+\sigma_{b}^{2}}} \exp \left[-\frac{\left(n_{t, \imath}-\mu_{s}-\mu_{b}\right)^{2}}{2\left(\sigma_{s}^{2}+\sigma_{b}^{2}\right)}\right] \\
& \times \frac{1}{\sqrt{\sigma_{b}^{2}}} \exp \left[-\frac{\left(n_{b, \imath}-\mu_{b}\right)^{2}}{2 \sigma_{b}^{2}}\right] .
\end{aligned}
$$

The probability distribution $P(\vec{x} \mid \Delta m)$ can be thought of as the probability for the number of counts in the signal region, the first term in Eq. 3. weighted by a prior given by the number of background counts, the second term in Eq. 3

Equation 3 is a general formula that can be used to estimate the mean and the variance of the satellite probability distribution, independent of the number of primaries that contribute to the sample. We integrate Eq. 3] and 
marginalize over the background mean and variance to obtain the probability for $\mu_{s}$ and $\sigma_{s}$. In our results below, error bars are derived as the area centered on the mean containing $68 \%$ of the cumulative probability distribution for $\mu_{s}$. We use uniform priors on the parameters that we estimate, $P(\vec{x})=$ const.

Assuming that the respective number counts $n_{t, \imath}$ and $n_{b, \imath}$ for a given primary reflect the mean of an underlying redshift probability distribution, Eq. 3 follows directly from the central limit theorem. This is explicitly true in the case of method 2, which makes the replacements $n_{t, \imath} \rightarrow\left\langle n_{t, \imath}\right\rangle$ and $n_{b, \imath} \rightarrow\left\langle n_{b, \imath}\right\rangle$ in Eq. 3. In the limit of a large number of primaries, we have verified that Eq. 3 reproduces the true mean of the full satellite probability distribution, as determined in Liu et al. (2011).

\section{RESULTS}

We begin by comparing the results of the various methods for estimating the number of satellites for several samples of host galaxies. The four panels in Figure 1 show the mean number of satellites around MW-like primaries that are brighter than $\Delta m$ magnitudes fainter than the primary, $\mu_{s}(<\Delta m)$, for the three methods described above. In each of the four panels, there is a different threshold cut on the magnitude of signal and background galaxies for the two methods that use photometric redshifts (methods 2 and 3 ). From left to right, the magnitude cuts on signal and background galaxies are $r<17.8,18.8,19.8,21.8$. Results from the spectroscopic analysis (method 1) are shown as blue diamonds in all panels; by definition this method only includes galaxies in the signal and background regions with $r<17.77$. Results from the full $p(z)$ sampling analysis (method 2 ) are shown as red triangles, and from the cut on mean photometric redshifts (method 3) are shown as black squares.

Figure 1indicates that for satellites with $r<17.8$ (leftmost panel), we find good agreement between all three methods, with large error bars for the method using simple $p(z)$ cuts. For these primaries, the $p(z)$ distribution is determined by galaxies in the main sample of SDSS, so the training sample is expected to be fully representative. The agreement between methods 1 and 2 is maintained for $\Delta m=4$ and $r<18.8$ (left two panels). However, we find that this agreement weakens for $\Delta m=5,6$, where the mean abundances as determined from method 2 underestimate the results from the spectroscopic analysis. This underestimation is most evident in the right panel of Fig. 1, and as discussed above most likely indicates that galaxies with $r>18.8$ and $z \lesssim 0.055$ are not adequately represented in the training sets. In addition, as expected, method 3 tends to overestimate the mean abundance for all $\Delta m$ and all magnitude limits. This is true both in the regime in which $\left\langle z_{p}\right\rangle_{90}=0.2$, for satellites with $r<19.8$ (left three panels), and $\left\langle z_{p}\right\rangle_{90}=0.5$, and for satellites with $r<21.8$ (right panel). These results indicate that the photometric redshift distributions for satellites dimmer than $r=17.8$ are likely biased towards higher redshifts, as a result of increasing incompleteness in the very lowest redshift galaxies in the training sample for dimmer galaxies.

What leads to this bias in the photometric redshifts, and is it avoidable? First, it is important to realize that our regime of interest is very low redshift, $z<0.02$, well outside the redshift range where photometric redshifts are generally used and tested. The $p(z)$ distributions calculated by Sheldon et al. (2011) use training sets from SDSS (Aihara et al. 2011), PRIMUS (Coil et al. 2011), zCOSMOS (Lilly et al. 2007), 2SLAQ (Cannon et al. 2006), VVDS (Garilli et al.|2008), DEEP2 (Weiner et al. 2005), CNOC2 (Yee et al. 2000), CFRS (Lilly et al. 1995), and TKRS (Wirth et al. 2004). For galaxies in our main regime of interest, $17.8<r<21.8$, the training sample is dominated by PRIMUS (Coil et al. 2011, , Cool et al, in preparation), which covers more than 9 sq. degrees to a depth of $i_{\mathrm{AB}} \sim 23.5(5.2 \mathrm{sq}$. degrees are included in the current training set). For PRIMUS, which is focused on science at $z>0.2$, there are at least two distinct issues that impact our analysis. First, the 4000 Åbreak falls out of the wavelength regime for $z<0.2$; without this feature the low-redshift spectra were more likely to be assigned a lower confidence flag $(Q=3)$ and were excluded in the $p(z)$ estimation (Carlos Cunha, private communication). Second, the analysis pipeline does not even attempt to measure accurate redshifts in the regime of our primaries $(z<0.02)$ because of the limited velocity resolution of the low-dispersion prism used by PRIMUS (John Moustakas \& Alison Coil, private communication). Both of these will impact the completeness of the low redshift sample and are likely to bias the $p(z)$ distribution against the redshift range of our primary samples.

One might also consider whether the biased $p(z)$ distribution could be avoided with another photometric redshift code. However, this range is challenging for any algorithm. For example, template-fitting codes without priors have known failure modes at very low redshift (Mandelbaum et al. 2008), and this would likely contaminate the low redshift sample.

From the information in Figure 1 we are able to obtain our best estimate for $\mu_{s}(<\Delta m)$ over the entire range of $\Delta m$. Specifically for our best estimate of $\mu_{s}(<\Delta m)$ we use the spectroscopic results for $\Delta m \leq 6$, and the results from method 3 for $\Delta m>6$. Figure 2 shows these as the main results of our analysis, in comparison to the observational points from the MW and M31. The results from method 3 are explicitly shown in the 6th column of Table 1, and the results from method 1 are shown in the last column of Table 1. Note that for the cases with a small number of primaries, which for our MW sample corresponds to $\Delta m>7$, only an upper limit on the mean can be determined.

Lares et al. (2011) report that the satellite population is uncertain in the central $100 \mathrm{kpc}$ around primary galaxies due to contamination from features in the extended halos of primaries. We have directly examined the images of many nearby, bright primaries and find that generally this systematic is accounted for in the Sheldon et al. (2011) galaxy sample used in our analysis. In addition we have explicitly examined how our results are affected when excluding the $100 \mathrm{kpc}$ around primaries, and we generally find minimal changes relative to those results presented in Fig. 2. For example, for Fornax-magnitude satellites around MW-like primaries, we find that the $90 \%$ c.l. upper limit on the mean reduces from 13 to 9 when excluding the central $100 \mathrm{kpc}$.

In addition to the distribution of the mean number of satellites, from our likelihood analysis we are able to esti- 

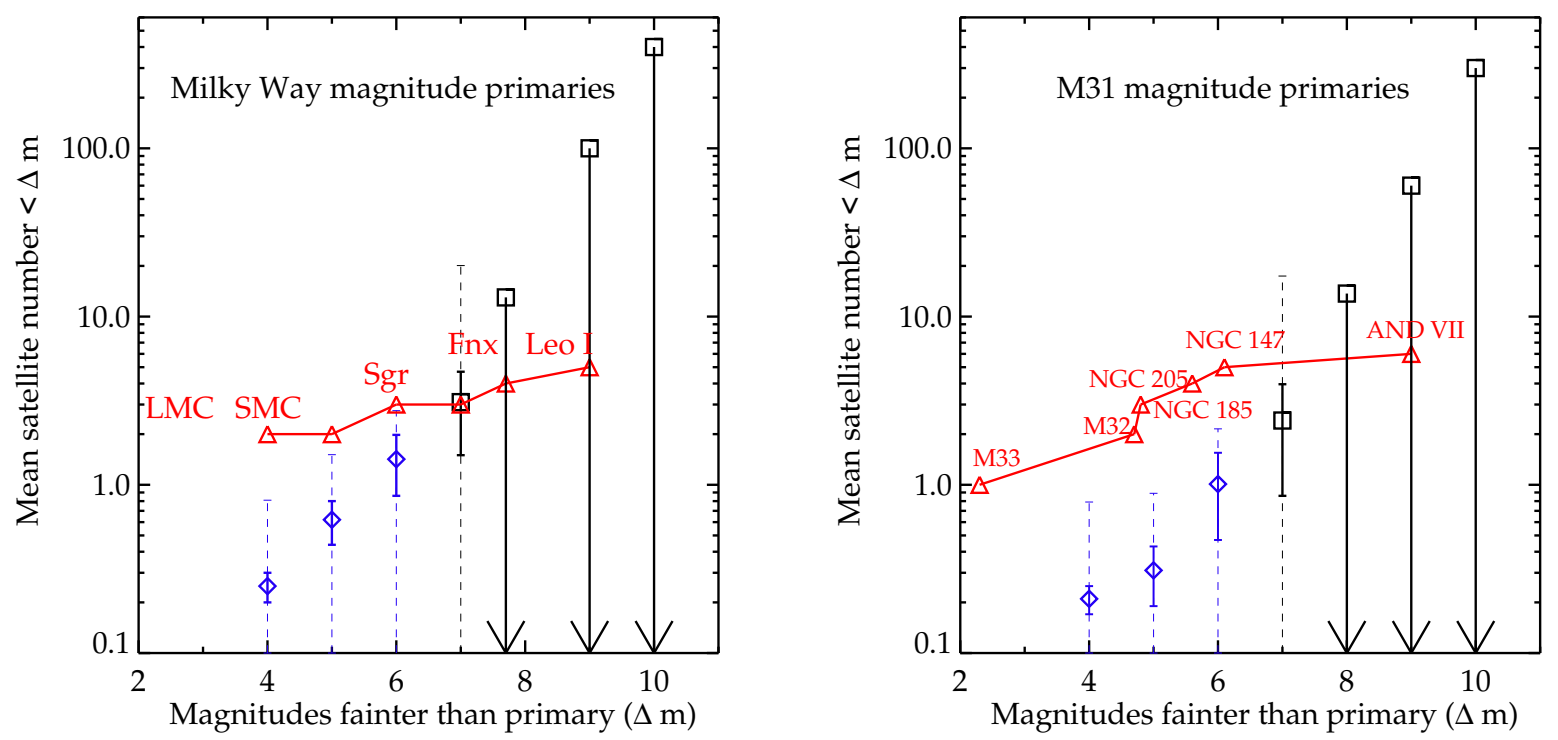

Figure 2. Left: Mean number of satellites brighter than $\Delta m$ magnitudes fainter than the primary galaxy, assuming primaries within \pm 0.25 magnitudes of the Milky Way. Blue diamonds are determined from the spectroscopic sample of satellites (method 1), black squares from the photometric sample (method 3). The solid errors are the uncertainty on the mean, the thin, dashed errors are the intrinsic scatter ( $\sigma_{s}$ from Eq. 3). The arrows indicate $90 \%$ c.l. upper limits. The red triangles indicate the Milky Way satellites. Right: Same as left, except for primaries within \pm 0.25 magnitudes of M31.

mate the intrinsic scatter, or $\sigma_{s}$, for each magnitude bin. As for the mean, $\sigma_{s}$ is determined from the full probability density distribution using Eq. 3. As an example for MW-like primaries and $\Delta m=[4,5]$, we find a mean intrinsic scatter of $\sigma_{s}=[0.56 \pm 0.04,0.89 \pm 0.19]$, where the errors represent one-sigma uncertainties as above. The best-fitting values for $\sigma_{s}$ are shown as thin, dashed error bars in Fig. 2 for $\Delta m \leq 7$. Via the method outlined in Liu et al. (2011), we are also able to estimate the full probability distribution down to $\Delta m=5$; here we find that the probability to obtain $[0,1,2,3,4]$ satellites with $\Delta m<5$ is $[0.59,0.25,0.11,0.03,0.02]$. Down to fainter magnitudes, the spectroscopic sample is too sparse to measure the full satellite probability distribution. These results indicate that there is still substantial intrinsic scatter in the satellite population, even at the brightest scales.

We note that the limits we present are strictly valid over the regime of surface brightness where the SDSS DR8 data is complete. For galaxies with half-light surface brightness $\lesssim 22.5 \mathrm{mag} / \mathrm{acrmin}^{2}$, which is the surface brightness of Leo I, the SDSS spectroscopic data is $\sim 90 \%$ complete (Blanton et al. 2005). For galaxies with surface brightness similar to Fornax or Sculptor, $\lesssim 23.5 \mathrm{mag} /$ acrmin $^{2}$, the surface brightness completeness is $\sim 50 \%$. We note that the surface brightness incompleteness has not been estimated directly for the photometric sample, but the agreement our results between the two sets of samples indicates that the issues may be of similar magnitude. We can get an estimate as to how the surface brightness incompleteness affects our results by comparing the integrated luminosity function of Blanton et al. (2005) that is corrected for incompleteness, as compared to the measured luminosity function. Down to the magnitude of Fornax, for example we find that the luminosity functions differ by a factor $\lesssim 2$ going to down surface brightness of $24 \mathrm{mag} / \mathrm{acrmin}^{2}$. If interested in constraining the population of objects down to this surface brightness, this factor should be taken as a conservative correction to the limits that are presented in Fig. 2. Thus in order to obtain many more bright satellites than are observed in the MW, it is clear that these satellites must have surface brightness much dimmer than the known bright MW satellites.

\section{COMPARISON TO PREVIOUS RESULTS}

There have been several recent analyses on the population of bright satellites around MW-analog galaxies along the lines presented in this paper. It is instructive to compare the results presented here to these previous analyses.

Guo et al. (2011) used SDSS DR7 to construct the luminosity function of satellites down to the magnitude scale of Fornax, correcting for the incompleteness of SDSS. These authors used best-fitting photometric redshifts from DR7 to eliminate obvious background galaxies. Our analysis differs from these authors in that we utilize both DR8 imaging and a maximum likelihood method that incorporates full photometric redshift probability distributions. We also directly quantify the bias in abundance counts for faint satellites that is incurred when utilizing available photometric redshifts. Via somewhat different methods for cutting background galaxies, Lares et al. (2011) use DR7 data to obtain a mean number of satellites down to the magnitude of Sagittarius for projected radii $\gtrsim 100 \mathrm{kpc}$. As we discuss above, we have verified that our results are consistent with these authors over the radial range considered, and further that we do not incur a significant bias by including galaxies within projected radii $<100 \mathrm{kpc}$. Tollerud et al. (2011) utilize the DR7 volume-limited spectroscopic sample and find that $\sim 40 \%$ of MW-analogs have satellites brighter than 
the LMC within $250 \mathrm{kpc}$. James \& Ivory (2011) use $\mathrm{H} \alpha$ narrow band imaging to search for start forming galaxies around 143 spiral galaxies like the MW, and find that nearly two-thirds do not have satellites that resemble the Magellanic Clouds. These latter two results are consistent with the spectroscopic results that we present for bright satellites.

\section{DISCUSSION AND CONCLUSION}

We have used DR8 photometric redshift data to limit the mean number of satellites around MW-analog galaxies down to ten magnitudes fainter than the MW. At least down to the scale of Sagittarius, the results indicate that the MW is not a significant statistical outlier in its number of bright, classical satellites.

Our $90 \%$ c.l. upper bound of $\lesssim 13$ satellites brighter than the Fornax dSph already places a strict bound on the efficiency of galaxy formation at the dSph luminosity scale. This is particularly true considering that there are anywhere from $\sim 25-75$ dark matter subhalos in the Aquarius simulations (Springel et al. 2008) that have present-day circular velocities greater than that of Fornax. Surface brightness incompleteness could increase this number, but likely not enough to bring it into agreement with predictions for the number of dense satellites in simulations. However, it is very interesting to note that the observational result we present is perfectly consistent with abundance matching extrapolations for the satellite luminosity function, which predict $\sim 1.2,1.7$ satellites for magnitude differences $\Delta m=7,10$ (Busha et al. 2011). This does not guarentee that such models will have the correct velocity function; in fact it appears increasingly difficult to simultaneously match both the luminosities and velocities of all of the satellites down to the Fornax scale.

In the future it will be exceedingly important to increase the sample of primary galaxies around which it is possible to measure satellites as faint as Fornax. Measuring the magnitude distribution at this faint scale will go a long way towards determining if the mapping between bright dSphs and dark matter subhalos is revealing the presence of detailed baryonic physics not yet accounted for in numerical simulations (Parry et al. 2011) or about the properties of dark matter (Lovell et al. 2011).

The principle uncertainty in accurately determining the satellite distribution from the SDSS photometric sample is the fidelity of the photometric redshifts. We have shown that our method using the full photometric redshift distribution is in excellent agreement with a method that directly uses spectroscopic redshifts for bright satellites where the training sample is representative. However, this method is biased to lower satellite numbers for dimmer magnitudes, which is an indication that the spectroscopic training samples used to construct the photometric redshift distributions are systematically missing the lowest redshift galaxies. Our measurements would be substantially improved if these samples were unbiased, as this would allow us to use the $p(z)$ method for a substantially larger number of host galaxies. It may be possible in the very near future to extend the training sample to include data from GAMA 2 , extending to $r<19.8$, which would allow us to extend our measure-

\footnotetext{
${ }^{2}$ http://www.gama-survey.org/
}

ment using the $p(z)$ method to the Fornax scale. With some care, it may also be possible to refine and improve the PRIMUS redshift finder in the range relevant to our low-redshift primaries $(z<0.02)$, thereby providing a more reliable photometric redshift training sample.

Given forthcoming data sets it will also be possible to significantly increase the number of primary galaxies; for example, the Dark Energy Survey (DES) 3 will produce a survey over 5000 sq. degrees and will observe galaxies to 24.3 in the $\imath$-band. If one considers satellites down to $r=24$, the DES is expected to identify more than 4000 primaries with satellites to Fornax magnitude differences, about 1600 primaries with Leo I-like satellites, and nearly 100 primaries with satellites as dim relative to their primary as Sculptor is to the Milky Way. At the Fornax scale, the statistics should be large enough to get a solid measurement using background subtraction even without robust photometric redshifts. The analysis here indicates that the primary challenge for the dimmest satellites around these primaries will be determining their redshift distribution.

In the future it will also be important to obtain kinematics on spectroscopically-confirmed satellites, as well as those that have a high probability to be satellites from their photometric redshifts. Comparing the velocity dispersion of these satellites to the $\sim 10 \mathrm{~km} / \mathrm{s}$ velocity dispersions of the MW dSphs will allow for a determination of both the luminosity function and the mass function of satellites down to the scale of classical dSphs. This is only currently possible with the sample of MW dSphs.

\section{ACKNOWLEDGEMENTS}

We thank Carlos Cunha for extensive discussions about photometric redshifts and comments on a draft, Michael Blanton for helpful discussions about the spectroscopic sample and surface brightness completeness, Erin Sheldon for help with edge effects and completeness issues for the photometric catalog, Brian Gerke and Lulu Liu for providing code from our previous work, John Moustakas and Alison Coil for helpful discussions about the PRIMUS catalog at low redshift, Marla Geha for many helpful discussions, and Michael Busha and Beth Willman for helpful comments on a draft. LES and RHW were supported by the National Science Foundation under grant NSF AST-090883. Part of this work was completed at the Aspen Center for Physics, with support from the National Science Foundation under Grant No. 1066293.

Funding for the NASA-Sloan Atlas has been provided by the NASA Astrophysics Data Analysis Program (08ADP08-0072). Funding for SDSS-III has been provided by the Alfred P. Sloan Foundation, the Participating Institutions, the National Science Foundation, and the U.S. Department of Energy. The SDSS-III web site is http://www.sdss3.org. SDSS-III is managed by the Astrophysical Research Consortium for the Participating Institutions of the SDSS-III Collaboration including the University of Arizona, the Brazilian Participation Group, Brookhaven National Laboratory, University of Cambridge, University of Florida, the French Participation Group, the German Participation Group, the Instituto de Astrofisica de Canarias, the Michigan State/Notre

\footnotetext{
${ }^{3}$ http://www.darkenergysurvey.org/
} 
Dame/JINA Participation Group, Johns Hopkins University, Lawrence Berkeley National Laboratory, Max Planck Institute for Astrophysics, New Mexico State University, New York University, Ohio State University, Pennsylvania State University, University of Portsmouth, Princeton University, the Spanish Participation Group, University of Tokyo, University of Utah, Vanderbilt University, University of Virginia, University of Washington, and Yale University. The Galaxy Evolution Explorer (GALEX) is a NASA Small Explorer. The mission was developed in cooperation with the Centre National d'Etudes Spatiales of France and the Korean Ministry of Science and Technology.

\section{REFERENCES}

Aihara, H., et al. 2011, Astrophys.J.Suppl., 193, 29

Benson, A. J., Lacey, C., Baugh, C., Cole, S., \& Frenk, C. 2002, Mon.Not.Roy.Astron.Soc., 333, 156

Blanton, M. R., Lupton, R. H., Schlegel, D. J., Strauss, M. A., Brinkmann, J., Fukugita, M., \& Loveday, J. 2005, Astrophys. J. , 631, 208

Boylan-Kolchin, M., Bullock, J. S., \& Kaplinghat, M. 2011, Mon.Not.Roy.Astron.Soc., 415, L40

Boylan-Kolchin, M., Springel, V., White, S. D., \& Jenkins, A. 2010, Mon.Not.Roy.Astron.Soc., 406, 896

Bullock, J. S., Kravtsov, A. V., \& Weinberg, D. H. 2000, Astrophys.J., 539, 517

Busha, M. T., Wechsler, R. H., Behroozi, P. S., Gerke, B. F., Klypin, A. A., et al. 2011, Astrophys. J., in press

Cannon, R., et al. 2006, Mon. Not. R. Astron. Soc., 372, 425

Chen, J., Kravtsov, A. V., Prada, F., Sheldon, E. S., Klypin, A. A., et al. 2006, Astrophys.J., 647, 86

Coil, A. L., Blanton, M. R., Burles, S. M., Cool, R. J., Eisenstein, D. J., et al. 2011, Astrophys.J., 741, 8

Cooper, A., Cole, S., Frenk, C., White, S., Helly, J., et al. 2010, Mon.Not.Roy.Astron.Soc., 406, 744

di Cintio, A., Knebe, A., Libeskind, N. I., Yepes, G., Gottlöber, S., \& Hoffman, Y. 2011, Mon. Not. R. Astron. Soc., 417, L74
Diemand, J., Kuhlen, M., Madau, P., Zemp, M., Moore, B., et al. 2008, Nature, 454, 735

Font, A. S., et al. 2011, Mon. Not. R. Astron. Soc., 417, 1260

Garilli, B., et al. 2008, Astron.\&Astrophys, 486, 683

Guo, Q., Cole, S., Eke, V., \& Frenk, C. 2011, Mon. Not. R. Astron. Soc., 417, 370

James, P. A., \& Ivory, C. F. 2011, Mon. Not. R. Astron. Soc., 411,495

Kleyna, J. T., Geller, M. J., Kenyon, S. J., \& Kurtz, M. J. 1997, Astron. J., 113, 624

Lares, M., Lambas, D. G., \& Domínguez, M. J. 2011, Astron. J., 142,13

Lilly, S., et al. 2007, Astrophys.J.Suppl., 172, 70

Lilly, S. J., Le Fevre, O., Crampton, D., Hammer, F., \& Tresse, L. 1995, Astrophys. J. , 455, 50

Liu, L., Gerke, B. F., Wechsler, R. H., Behroozi, P. S., \& Busha, M. T. 2011, Astrophys. J., 733, 62

Lovell, M., et al. 2011, arXiv:1104.2929

Mandelbaum, R., Seljak, U., Hirata, C., Bardelli, S., Bolzonella, M., et al. 2008, Mon.Not.Roy.Astron.Soc., 386, 781

Parry, O. H., Eke, V. R., Frenk, C. S., \& Okamoto, T. 2011, arXiv:1105.3474

Sheldon, E. S., Cunha, C., Mandelbaum, R., Brinkmann, J., \&

Weaver, B. A. 2011, arXiv:1109.5192

Somerville, R. S. 2002, ApJ, 572, L23

Springel, V., et al. 2008, Mon. Not. R. Astron. Soc., 391, 1685

Strigari, L. E., Frenk, C. S., \& White, S. D. M. 2010, Mon. Not. R. Astron. Soc., 408, 2364

Tollerud, E. J., Boylan-Kolchin, M., Barton, E. J., Bullock, J. S., \& Trinh, C. Q. 2011, Astrophys.J., 738, 102

van den Bergh, S. 2000, PASP, 112, 529

Wadepuhl, M., \& Springel, V. 2011, Mon. Not. R. Astron. Soc., 410, 1975

Walsh, S. M., Willman, B., \& Jerjen, H. 2009, Astron. J., 137, 450

Weiner, B. J., Phillips, A. C., Faber, S., Willmer, C. N., Vogt, N. P., et al. 2005, Astrophys.J., 620, 595

Willman, B. 2010, Advances in Astronomy, 2010

Wirth, G. D., Willmer, C. N., Amico, P., Chaffee, F. H., Goodrich, R. W., et al. 2004, Astron.J., 127, 3121

Yee, H., Morris, S., Lin, H., Carlberg, R., Hall, P., et al. 2000, Astrophys.J.Suppl., 129, 475 